\title{
Evaluation of vascular quality of life questionnaire in dialysis patients with peripheral arterial disease treated by low-density lipoprotein apheresis
}

Kojiro Nagai ${ }^{{ }^{* \dagger}}$, Megumi Harada ${ }^{2+}$, Narushi Yokota ${ }^{3}$, Daigo Okada $^{3}$, Hiroyuki Michiwaki $^{2}$, Daisuke Hirose $^{2}$, Toshio Doi ${ }^{1}$, Jun Minakuchi ${ }^{3}$ and Kenji Tsuchida ${ }^{3^{*}}$

\begin{abstract}
Background: Peripheral arterial disease (PAD) is a common complication in dialysis patients. Early diagnosis and treatment are recommended. Low-density lipoprotein apheresis (LDL-A) is a potential therapy to improve PAD. However, the mechanism has yet to be fully clarified due to lack of established quantitative methods to assess the therapeutic effects of LDL-A treatment. Improvement of skin perfusion pressure (SPP) or ankle brachial index (ABI) is a representative therapy goal, but clinical symptoms were not always consistent with the values of SPP/ABI. Vascular quality of life questionnaire (VascuQOL) was proposed as a disease-specific QOL score, getting validated recently. The possibility of VascuQOL to reflect the severity of PAD in dialysis patients and evaluate the therapeutic effects of LDL-A has yet to be elucidated.
\end{abstract}

Methods: This is an observational study. LDL-A treatment was performed in 32 dialysis patients with PAD. They were divided to critical limb ischemia (CLI) group (17 subjects) and non-CLI group (15 subjects) according to their clinical manifestations. We examined the relationship of PAD severity with SPP, ABI, VasCUQOL, and lipid profile such as apoB/apoA-I ratio, malondialdehyde-modified LDL, and remnant-like particles cholesterol. Furthermore, we evaluated these parameters successively to find out a suitable therapeutic marker just after the first LDL-A, at tenth LDL-A, and 1 month after completion of LDL-A treatment.

Results: All of the lipid markers were higher in CLI patients, but not significantly different from those in the non-CLI group. They decreased significantly just after LDL-A, although no changes were observed 1 month after completion of LDL-A treatment. ABI was significantly different between the CLI and non-CLI groups, but did not improve by LDL-A treatment. By contrast, SPP was ameliorated significantly and the peak was at tenth LDL-A. Among VascuQOL domains, "Symptom" and "Emotional" domains were significantly different between the CLI and non-CLI groups. The average score of VascuQOL increased successively until 1 month after completion of LDL-A treatment.

Conclusions: Several domains of VascuQOL can reflect the severity of PAD in dialysis patients. VascuQOL was a useful marker to show the prolonged therapeutic effects of LDL-A treatment in dialysis patients with PAD, independent of SPP.

Keywords: Vascular quality of life questionnaire (VascuQOL), Peripheral arterial disease (PAD), Low-density lipoprotein apheresis (LDL-A), Skin perfusion pressure (SPP), Ankle brachial index (ABI)

\footnotetext{
*Correspondence: knagai@tokushima-u.ac.jp; ktsuchida@khg.or.jp

${ }^{\dagger}$ Equal contributors

'Department of Nephrology, Institute of Biomedical Sciences, Tokushima

University Graduate School, 3-18-15, Kuramoto-cho, Tokushima-shi,

Tokushima 770-8503, Japan

${ }^{3}$ Department of Kidney Disease, Kawashima Hospital, 1-39,

Kitasakoichiban-cho, Tokushima-shi, Tokushima 770-0011, Japan

Full list of author information is available at the end of the article
} 


\section{Background}

The prevalence and incidence of peripheral arterial disease (PAD) in dialysis patients are increasing worldwide. PAD can occur in dialysis patients with few symptoms, often confirmed only after the patient has already exhibited symptoms of critical limb ischemia (CLI) [1, 2]. Patients with CLI commonly have cardiovascular and cerebrovascular diseases. Since survival of patients with CLI is significantly worse than patients only with intermittent claudication, early diagnosis and treatment are quite important [3].

Generally, PAD in dialysis patients is resistant to drug treatment, and surgical intervention is difficult. Thus, low-density lipoprotein apheresis (LDL-A) is one of the potential therapies for PAD to improve symptoms and wound healings [4]. LDL-A can show "short-term" effects on lipid profile just after the therapy. However, the quantitative evaluation methods of LDL-A "longterm" effects at last session of one full course LDL-A therapy and "prolonged" effects several months after LDL-A therapy completion have not been established. Skin perfusion pressure (SPP) and ankle brachial index (ABI) are used to detect PAD in dialysis patients and can be useful markers to choose LDL-A therapy responders. Especially, SPP was reported to be more accurate to detect PAD in dialysis patients than ABI [5]. However, the obstruction or improvement of peripheral circulation is not always parallel to patients' symptom $[5,6]$. Kobayshi summarized the several mechanisms of LDL-A [7]: (1) direct effect of lowered lipids or reduction of lipid toxicity; (2) improvement of hemorpheology by removing fibrinogen; (3) vasodilatory effects by an increase in VEGF/NO/bradykinin production/endothelium-derived vasodilatory factors or IGF-I or a decrease in thromboxane A2 [8,9]; (4) reduction of circulating vascular permeability factors; (5) anti-inflammatory effects-reduction of oxidized LDL, P-selectin, CRP, and ICAM-1 [10, 11]; (6) improvement of endothelial dysfunction [12]; and (7) enhancement of corticosteroids or cyclosporine response by changing drug interactions following lipid removal. However, the point is that we have not found a definite quantitative marker to suggest the long-term and prolonged therapeutic effects of LDL-A treatment on PAD in dialysis patients.

Vascular quality of life questionnaire (VascuQOL) was presented by Morgan et al. to assess the quality of life in PAD patients [13]. The utility of this disease-specific questionnaire was evaluated elsewhere $[14,15]$. However, to our knowledge, the utility of VascuQOL in dialysis patients with PAD has yet to be fully elucidated.

Therefore, in this study, we examined the relationship of peripheral circulation, VascuQOL, and lipid profiles with PAD severity to pursue an effective screening method that reflects the severity of PAD in dialysis patients. We also investigated the changes of these quantitative parameters by LDL-A treatment to find out a novel marker to show the long-term and prolonged effects of LDL-A treatment in dialysis patients with PAD.

\section{Methods \\ Design and subjects}

This study included 32 dialysis patients with PAD (24 male and 8 female patients aged 51 to 85 years) who underwent LDL-A treatment since May 2013. Critical limb ischemia (CLI) was defined, per "ACC/AHA Guidelines for the Management of Patients with PAD" [16] and concordant TASC-II PAD guidelines [3], in patients with Fontaine stages III and IV manifestations such as ischemic rest pain and minor or major tissue loss of greater than 2 weeks duration. Thus, the patients were divided into the CLI (15 subjects) and non-CLI (17 subjects with intermittent claudication, Fontaine stage II) groups (Table 1).

LDL-A was performed once a week on the day between the first and second or second and third dialysis session of the week, as a rule, with 10 rounds of LDL-A set as a cycle to be provided over the course of approximately 2.5 months (Fig. 1). The mean plasma throughput was determined to be $2900 \mathrm{~mL}$ for one apheresis based on the treatment time and switchover timing of LDL absorption column, Liposorber LA-15 (Kaneka, Osaka, Japan). During LDL-A treatment, oral administration of vasodilator, anti-platelet, and anti-lipidemic drugs was performed as usual with no change, and the conditions of dialysis remained unchanged. In addition, our normal treatments were provided continuously for cutaneous wounds due to PAD, nail thickening, and foot care.

The aim of this study is to find the serological and clinical quantitative tests which can have the relationship with PAD severity or suggest the short-, long-term, and prolonged effects of LDL-A treatment. Therefore, several valuables described below were monitored repeatedly. The non-fasting blood samples for the serological tests were taken in the beginning of the first LDL-A (Fig. 1, point $\mathrm{A}$ ), just after the first LDL-A (Fig. 1, point $\mathrm{B}$ ), the beginning of tenth LDL-A (Fig. 1, point C), 1 month after completion of LDL-A treatment (Fig. 1, point D). The clinical quantitative tests were performed just before the first LDL-A (Fig. 1, point A), tenth LDL-A (Fig. 1, point C), and 1 month after completion of LDLA treatment (Fig. 1, point D). B, C, and D points were for evaluating the short- and long-term and prolonged effects of LDL-A treatment, respectively.

As the serological tests, apoB/apoA-I ratio was determined by turbidimetric latex agglutination assays (BML, Inc., Tokyo, Japan), reported to be a surrogate marker of PAD [17]. In addition, malondialdehyde-modified LDL 
Table 1 Clinical characteristics of the subjects enrolled

\begin{tabular}{|c|c|c|c|}
\hline & Non-CLI $(n=17)$ & $\mathrm{CLI}(n=15)$ & $P$ value \\
\hline Age (years) & $70.4 \pm 8.0$ & $72.0 \pm 7.6$ & 0.57 \\
\hline Gender (male/female) & $15 / 2$ & $9 / 6$ & 0.08 \\
\hline Body mass index $\left(\mathrm{kg} / \mathrm{m}^{2}\right)$ & $20.9 \pm 2.6$ & $23.4 \pm 3.7$ & 0.04 \\
\hline Diabetes mellitus ( $n, \%)$ & $11,64.7 \%$ & $15,100 \%$ & 0.01 \\
\hline Cardiovascular disease $(n, \%)$ & $14,82.4 \%$ & $14,93.3 \%$ & 0.35 \\
\hline Cerebrovascular disease $(n, \%)$ & $5,29.4 \%$ & $5,33.3 \%$ & 0.56 \\
\hline Duration of hemodialysis (years) & $10.6 \pm 9.1$ & $9.9 \pm 8.4$ & 0.98 \\
\hline \multicolumn{4}{|l|}{ Laboratory data } \\
\hline Total cholesterol (mg/dL) & $161.2 \pm 41.3$ & $178.5 \pm 46.3$ & 0.24 \\
\hline Triglyceride (mg/dL) & $98.1 \pm 78.5$ & $127.6 \pm 48.0$ & 0.03 \\
\hline High-density lipoprotein cholesterol (mg/dL) & $52.1 \pm 15.2$ & $52.1 \pm 14.0$ & 0.99 \\
\hline C-reactive protein (mg/dL) & $0.34 \pm 0.63$ & $0.44 \pm 0.66$ & 0.30 \\
\hline Albumin (g/dL) & $3.51 \pm 0.32$ & $3.43 \pm 0.28$ & 0.46 \\
\hline \multicolumn{4}{|l|}{ Medications } \\
\hline Statin $(n, \%)$ & $4,23.5 \%$ & $8,53.3 \%$ & 0.08 \\
\hline Erythropoietin, darbepoetin alfa $(n, \%)$ & $8,47.1 \%$ & $7,46.7 \%$ & 0.98 \\
\hline Erythropoietin, epoetin beta pegol $(n, \%)$ & $5,29.4 \%$ & $5,33.3 \%$ & 0.81 \\
\hline Anti-platelet $(n, \%)$ & $17,100 \%$ & $15,100 \%$ & N/A \\
\hline Warfarin $(n, \%)$ & $2,11.8 \%$ & $4,26.7 \%$ & 0.27 \\
\hline Anti-hypertensive, ARB ( $n, \%)$ & $2,11.8 \%$ & $1,6.7 \%$ & 0.55 \\
\hline Anti-hypertensive, Ca antagonist ( $n, \%)$ & $4,23.5 \%$ & $4,26.7 \%$ & 0.58 \\
\hline Anti-hypertensive, Beta blocker $(n, \%)$ & $3,17.6 \%$ & $5,33.3 \%$ & 0.27 \\
\hline Anti-hypertensive, Alpha blocker ( $n, \%)$ & $2,11.8 \%$ & $0,0 \%$ & 0.27 \\
\hline
\end{tabular}

$A R B$ angiotensin II receptor blocker, $C a$ calcium, N/A not applicable

(MDA-LDL) and remnant-like particle cholesterol (RLPC) were measured by an enzyme-linked immunosorbent assay (BML, Inc., Tokyo, Japan). MDA-LDL is a marker of coronary artery disease severity [18], which is an epitope of oxidized LDL, a key actor in the initiation and

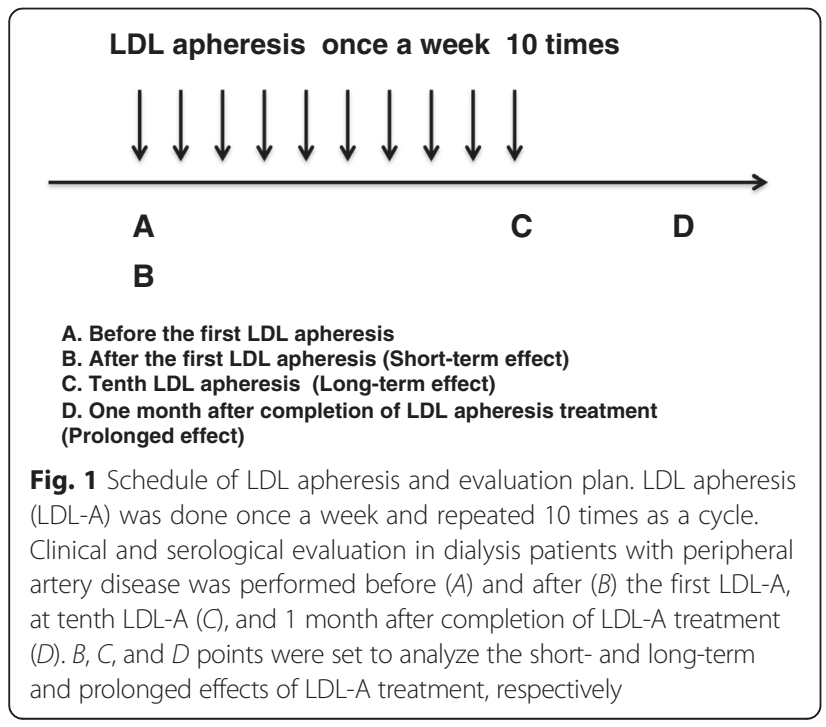

acceleration of atherosclerosis [19]. RLP-C is atherogenic fraction of triglyceride-rich lipoproteins, reportedly associated with the development of PAD [20, 21]. All of the items have not been investigated whether they can be a marker of the prolonged effects of LDL-A treatment in dialysis patients with $\operatorname{PAD}[9,22,23]$.

As the clinical quantitative tests, to evaluate the peripheral perfusion of the patients, ABI and SPP were monitored. ABI was measured in all patients by using an ABI-form (Colin, Tokyo, Japan) that simultaneously measured unilateral brachial pressure in the arm without an arteriovenous fistula and ankle blood pressure by using an oscillometric method. ABI was calculated as the ratio of ankle systolic pressure divided by brachial systolic pressure. For SPP, we used a Laser Doppler PAD3000 (Kaneka, Osaka, Japan) according to the method described by Okamoto et al. [5]. Briefly, the laser Doppler skin perfusion pressure transducer consists of a laser Doppler probe secured within the bladder of a blood pressure cuff that contains a transparent polyvinylchloride window so that microcirculatory perfusion measurements can be made during cuff deflation. The mean values obtained from both legs were applied for analysis. 


\section{VascuQOL}

In order to assess clinical symptoms quantitatively, we used Japanese version of VascuQOL, a disease-specific QOL score, validated previously [24, 25]. VascuQOL contains 25 questions measuring five domains of health status: Activity (8 questions), Symptom (4 questions), Pain (4 questions), Emotional (7 questions), and Social (2 questions). Each question has a 7-point response scale. The score ranges from 1 (worst QOL) to 7 (best QOL) [26]. Fourteen patients (82.4\%) in the non-CLI group and 8 patients $(53.3 \%)$ in the CLI group could complete all the questions at all of the three evaluation points.

\section{Statistical analysis}

All values are expressed as mean \pm SD. Statistical analysis was performed using SPSS for Windows version 13.0 (SPSS, Inc., Chicago, IL, USA). Baseline characteristics between CLI and non-CLI patients were compared using Student's $t$ test or Welch's $t$ test, if data were normally distributed. Non-normal data were analyzed by Man-Whitney's $U$ test. $F$ test was used for comparing the factors of total deviation. Prevalence data were analyzed by means of chi-square or Fisher's exact probability test. The changes of serological and clinical parameters in the time course of LDL-A treatment were analyzed using paired $t$ test or Wilcoxon signed-ranks test, as appropriate. Receiver operating characteristic (ROC) curve analysis was performed to assess sensitivity or specificity. Significance was defined by $P$ less than 0.05 .

\section{Results}

\section{Clinical and histological characteristics}

The patient background was as follows. The 32 subjects included 26 subjects with diabetes (81.3\%), 28 with cardiovascular disease (87.5\%), 10 with cerebrovascular disease (31.3\%), and 5 with smoking (15.6\%), showing that many of them exhibited complications of diabetes and vascular disease. Table 1 presents the background of the 17 subjects in the non-CLI group (53.1\%) and 15 subjects in the CLI group (46.9 \%). The CLI group had higher body mass index and serum triglyceride values with a larger percentage of DM patients than the nonCLI group. In regard to medication, anti-platelet drug was administered to all of the participants.

\section{Lipid profile showed the short-term improvement by LDL-A}

First, in the beginning of the first LDL-A, all of the apoB/apoA-I ratio, MDA-LDL, and RLP-C values were not statistically different between the CLI and non-CLI groups.

Next, lipid profile was followed until 1 month after completion of LDL-A treatment. In both groups, the values of apoB/apoA-I ratio after the first LDL-A and in the beginning of tenth LDL-A were significantly lower than those in the beginning of the first LDL-A. However, apoB/apoA-I ratio measured 1 month after completion of LDL-A treatment was equivalent to the level in the beginning of the first LDL-A (Fig. 2a). MDA-LDL decreased significantly after the first LDLA. However, there was no significant difference between the beginning of the first and tenth LDL-A (Fig. 2b). As for RLP-C, in the CLI group, a significant decrease was confirmed after the first LDL-A and in the beginning of tenth LDL-A, compared with that in the beginning of the first LDL-A, but no significant difference was found 1 month after completion of LDL-A treatment. In the non-CLI group, significant decrease was detected only just after the first LDL-A (Fig. 2c). Therefore, in this study, lipid profile improved for a short term by LDLA, but they were not useful to distinguish the CLI and non-CLI groups. Besides, LDL-A treatment did not ameliorate the lipid markers for a long term.

\section{Evaluation of peripheral circulation was useful to distinguish the severity of PAD and assess the long-term} therapeutic effects of LDL-A against PAD in dialysis patients $\mathrm{ABI}$ and SPP were also continuously monitored as representative tests for peripheral circulation. Before the first LDL-A, ABI showed a significant difference between the CLI and non-CLI groups, while SPP did not (Fig. 3a, b). In contrast, in the non-CLI group, SPP increased significantly at tenth LDL-A and was sustained until 1 month after completion of LDL-A treatment, though the values decreased after completion of LDL-A treatment (Fig. 3b). These findings suggest that, as reported, peripheral circulation monitoring can be a clue to distinguish the severity of PAD in dialysis patients and useful to evaluate the long-term therapeutic effects of LDL-A treatment.

\section{VascuQOL suggested the presence of CLI and reflected the prolonged therapeutic effects of LDL-A treatment in dialysis patients}

In this study, 22 patients $(68.8 \%$, called VascuQOL group) could reply to all the questions required. Their basic characteristics and the average scores of VascuQOL were shown in Table 2. Before the first LDL-A, the average score of VascuQOL was lower in the CLI group than that in the non-CLI group, but not reaching statistical significance (Fig. 4a, $P=0.07$ ). Considering each domain of health status, "Symptom" and "Emotional" were significantly lower in the CLI group than those in the non-CLI group (Fig. 4c, e). In ROC analysis, "Symptom" score less than 3.625 or "Emotional" score less than 3.929 indicated the presence of CLI with $75.0 \%$ sensitivity and $85.7 \%$ specificity. Area under the curve was 0.804 and 0.768 , respectively. After LDL-A started, the 


\section{a. ApoB/apoA-I ratio}

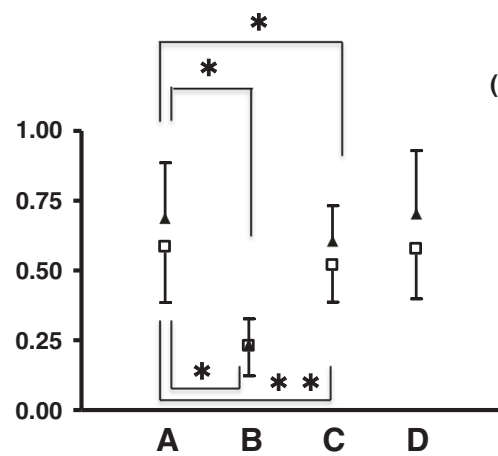

\section{RLP-C}

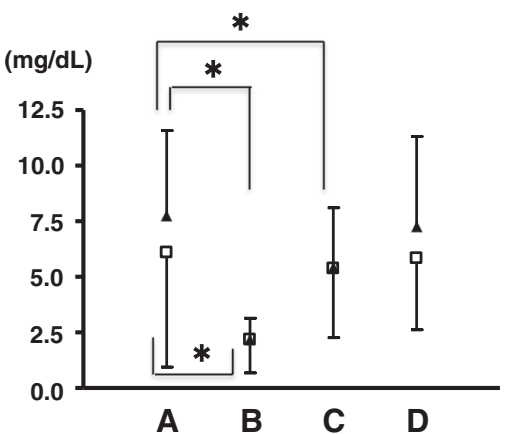

b. MDA-LDL

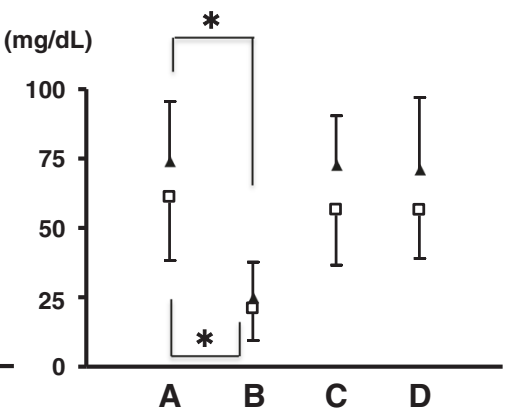

Non-CLI group

$\triangle$ CLI group

Fig. 2 Time course of lipid profile. White square shows the non-CLI group $(N=17)$. Black triangle represents the CLI group $(N=15)$. $A$ before the first LDL-A. $B$ after the first LDL-A:C at tenth LDL-A. D 1 month after completion of LDL-A treatment. a-c At starting point (point A), all of the apoB/apoA-I ratio, MDA-LDL, and RLP-C values were higher in the CLI group, but not statistically different between the CLI and non-CLI groups. a apoB/apoA-I ratio improved after the first $L D L$ apheresis $(L D L-A)$ and in the beginning of tenth $L D L-A$ significantly. However, it returned equivalent to the level of starting point 1 month after completion of LDL-A treatment. b MDA-LDL decreased significantly after the first LDL-A. However, there was no significant difference between the beginning of the first and tenth LDL-A. c As for RLP-C, in the CLI group, a significant decrease was confirmed after the first LDL-A and in the beginning of tenth LDL-A, compared with that in the beginning of the first LDL-A, but no significant difference was found 1 month after completion of LDL-A treatment. In the non-CLI group, a significant decrease was detected only just after the first LDL-A. All values are expressed as mean \pm SD. ${ }^{*} P<0.01 .{ }^{*} P<0.05$

average score of VascuQOL increased successively until 1 month after completion of LDL-A treatment, especially in the CLI group (Fig. 4a). In addition, "Symptom" and "Pain" improved in both groups, and "Activity" and "Emotional" increased significantly in the CLI group at 1 month after completion of LDL-A treatment (Fig. 4b-f). Among the VascuQOL group, SPP also improved significantly until 1 month after completion of LDL-A treatment in the non-CLI group, but the peak was at tenth LDL-A. Contrary to this, ABI rather got worse, especially in the CLI group. Apo B/apoA-I ratio, MDA-LDL, and RLP-C did not change at 1 month after completion of LDL-A treatment in the VascuQOL group (data not shown). These results show that VascuQOL is one of the promising quantitative methods to suggest the presence of CLI in dialysis PAD patients and evaluate the prolonged therapeutic effects of LDL-A treatment.

\section{Discussion}

In this study, we demonstrate that VascuQOL, a diseasespecific QOL score, can be the quantitative method to evaluate the prolonged therapeutic effects of LDL-A treatment on PAD in dialysis patients. This questionnaire can be useful for early detection of CLI in dialysis PAD patients. SPP was more preferable to detect the improvement of peripheral circulation by LDL-A treatment compared as ABI.

VascuQOL is getting noticed as a suitable diseasespecific QOL score in intermittent claudication and CLI. Among several disease-specific QOL instruments, VascuQOL and its domains were reportedly most responsive to treatment [27]. The score has been used in several prospective studies [28]. Mazai et al. showed that VascuQOL was correlated with clinical indicators of lower limb ischemia, such as treadmill walking distances and ABI. However, the degree of improvement 

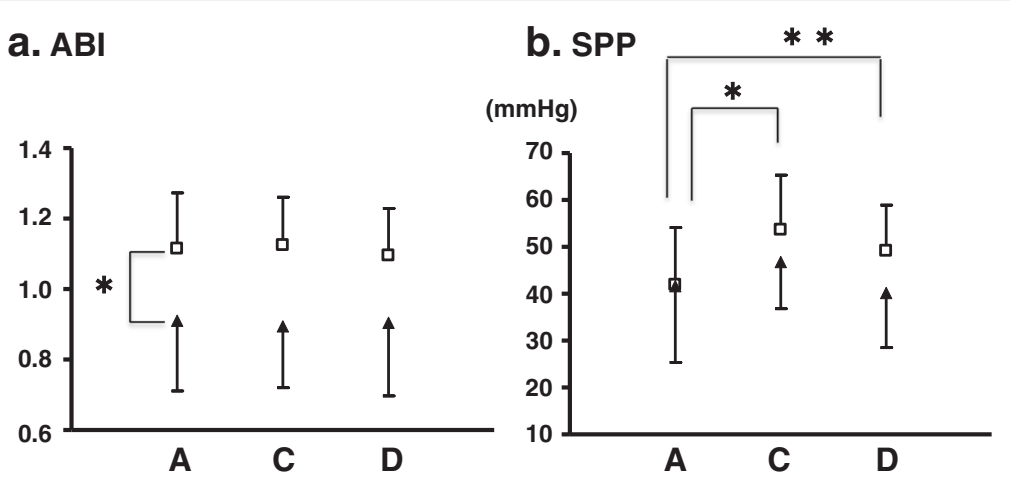

Fig. 3 Time course of peripheral circulation. White square shows the non-CLI group ( $N=17)$. Black triangle represents the $C L I$ group $(N=15)$. $A$ before the first LDL-A. C at tenth LDL-A. D 1 month after completion of LDL-A treatment. a, b At starting point (point A), ABI showed a significant difference between the CLI and non-CLI groups, while SPP did not. a ABI did not improve by LDL-A treatment. $\mathbf{b}$ SPP increased significantly at tenth LDL-A and was sustained until 1 month after completion of LDL-A treatment, though the values decreased after completion of LDL-A treatment in the non-CLI group. All values are expressed as mean \pm SD. ${ }^{*} P<0.01 .{ }^{* *} P<0.05$

Table 2 Basic characteristics of patients who completed VascuQOL

\begin{tabular}{|c|c|c|c|c|c|c|c|c|}
\hline & Age & Gender & $\begin{array}{l}D \\
M\end{array}$ & HD dur. & $\begin{array}{l}\text { Fontaine } \\
\text { stage }\end{array}$ & $\begin{array}{l}\text { VascuQOL } \\
\text { First LDL-A }\end{array}$ & $\begin{array}{l}\text { VascuQOL } \\
\text { Tenth LDL-A }\end{array}$ & $\begin{array}{l}\text { VascuQOL } \\
\text { One month }\end{array}$ \\
\hline \multirow[t]{14}{*}{ Non-CLI } & 68 & Male & $(-)$ & 21 & $\|$ & 4.24 & 4.12 & 2.36 \\
\hline & 66 & Male & $(+)$ & 14 & $\|$ & 5.36 & 5.28 & 5.44 \\
\hline & 74 & Male & $(+)$ & 4 & $\|$ & 5.68 & 6.24 & 6.24 \\
\hline & 74 & Male & $(+)$ & 6 & $\|$ & 5.44 & 5.6 & 5.84 \\
\hline & 73 & Male & $(+)$ & 7 & $\|$ & 3.88 & 4 & 4 \\
\hline & 80 & Male & $(-)$ & 5 & $\|$ & 2.36 & 2.88 & 2.56 \\
\hline & 51 & Male & $(-)$ & 9 & $\|$ & 4.72 & 4.48 & 5.48 \\
\hline & 70 & Male & $(+)$ & 1 & $\|$ & 5.72 & 5.88 & 5.36 \\
\hline & 73 & Male & $(+)$ & 7 & $\|$ & 5.56 & 6.04 & 5.76 \\
\hline & 63 & Male & $(+)$ & 20 & $\|$ & 1.64 & 3.04 & 2.96 \\
\hline & 68 & Female & $(+)$ & 12 & $\|$ & 4.6 & 5.36 & 5.52 \\
\hline & 68 & Male & $(+)$ & 1 & $\|$ & 5.92 & 5.4 & 5.48 \\
\hline & 78 & Male & $(-)$ & 22 & $\|$ & 5.84 & 5.88 & 5.44 \\
\hline & 70 & Male & $(+)$ & 1 & $\|$ & 6.04 & 6.24 & 6.48 \\
\hline \multirow[t]{8}{*}{$\mathrm{CLI}$} & 76 & Male & $(+)$ & 2 & III & 2.68 & 2.92 & 3.28 \\
\hline & 57 & Male & $(+)$ & 7 & IV & 2.64 & 4.6 & 4.16 \\
\hline & 67 & Male & $(+)$ & 12 & III & 3.36 & 2.52 & 3.64 \\
\hline & 72 & Female & $(+)$ & 9 & III & 1.92 & 1.8 & 2.84 \\
\hline & 81 & Male & $(+)$ & 1 & III & 2.24 & 3.04 & 3.72 \\
\hline & 67 & Female & $(+)$ & 16 & III & 3.36 & 3.48 & 3.64 \\
\hline & 70 & Male & $(+)$ & 21 & IV & 6.04 & 6.64 & 6.76 \\
\hline & 64 & Female & $(+)$ & 1 & IV & 5.28 & 4.76 & 5.16 \\
\hline
\end{tabular}



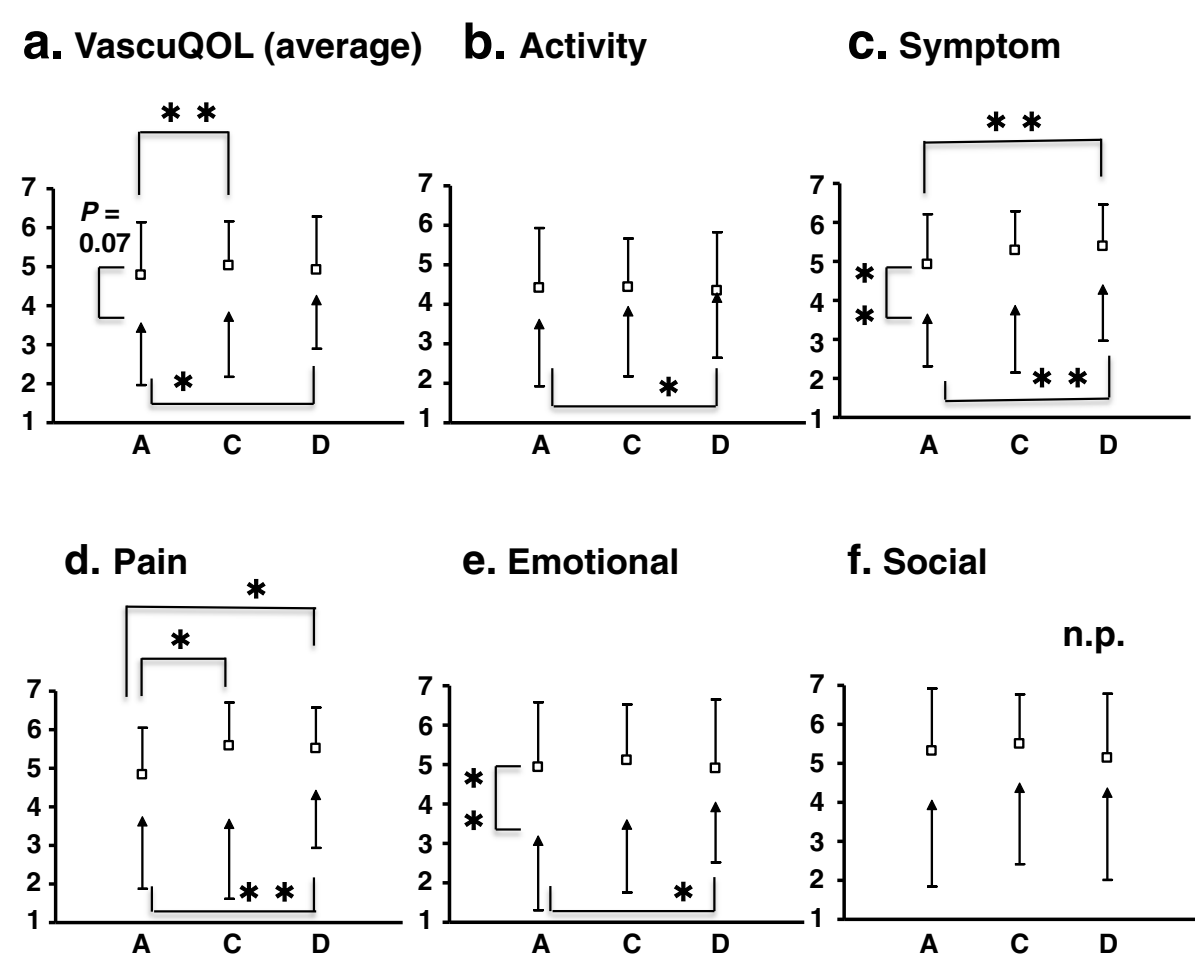

e. Emotional

f. Social

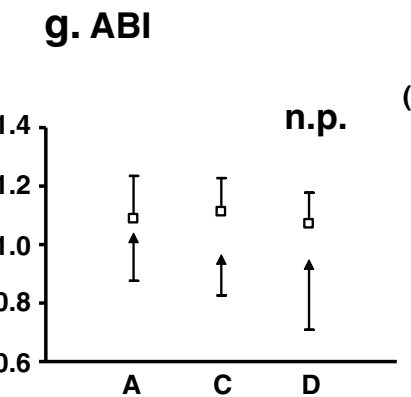

h. SPP

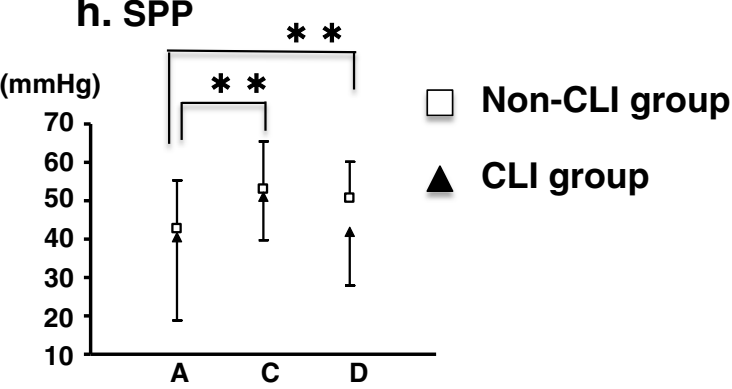

Fig. 4 Time course of VascuQOL and its domains. Among the VascuQOL group, white square shows the non-CLI group ( $N=14)$. Black triangle represents the $C L I$ group $(N=8)$. A before the first LDL-A. C at tenth $L D L-A . D 1$ month after completion of LDL-A treatment. a-f At starting point (point A), the average score of VascuQOL was lower in the CLI group, but not reaching statistical significance $(P=0.07)$. "Symptom" and "Emotional" were significantly lower in the CLI group than those in the non-CLI group. After LDL-A started, the average score of VascuQOL (a) increased successively until 1 month after completion of LDL-A treatment, especially in the CLI group. "Symptom" (c) and "Pain" (d) improved in both groups, and "Activity" (b) and "Emotional" (e) increased significantly in the CLI group at 1 month after completion of LDL-A treatment. $\mathbf{g}$, h Among the VascuQOL group, SPP (h) improved significantly until 1 month after completion of LDL-A treatment in the non-CLI group, but the peak was at tenth LDL-A. Contrary to this, $A B I(\mathbf{g})$ rather got worse, especially in the CLI group. All values are expressed as mean \pm SD. ${ }^{*} P<0.01 .{ }^{* *} P<0.05$

seen in clinical indicators of lower limb ischemia is not reflected in these scores. These findings support the use of composite outcome measures with independent assessment of QOL as an independent outcome measure in intervention studies in these patients [29]. However, to our knowledge, this questionnaire has not been applied to assess LDL-A treatment effects on PAD in dialysis patients. This is the first report to suggest its utility as a unique and independent method to quantify the therapeutic results of LDL-A treatment against PAD in dialysis patients. VascuQOL could detect the prolonged effects of LDL-A treatment and the time course of the score was not parallel to SPP, one of the representative clinical indicators. SPP could indicate the LDL-A effects at the last LDL-A, but not excellent to estimate the prolonged improvement by LDL-A treatment. We believe that quantitative evaluation using the combination of VascuQOL and SPP can be a novel marker in order to clarify the precise mechanism of LDL-A treatment.

As described in the "Background" section, early diagnosis of PAD is important to prevent the disease 
progression to cause CLI. Okamoto et al. demonstrated that SPP set at $50 \mathrm{~mm} \mathrm{Hg}$ was more accurate than ABI to detect PAD, with sensitivity of $84.9 \%$ and specificity of $76.9 \%$ [5]. In our study, SPP was not significantly different between the CLI group and non-CLI group. We do need the other screening method that reflects the severity of PAD in dialysis patients. Nguern et al. reported that patients with CLI have a low QOL at baseline [30]. VascuQOL consists of five domains of health status. In our study, among the domains, "Symptom" and "Emotional" showed significant differences between the CLI group and non-CLI group. ROC analysis showed that cutoff scores of "Symptom" or "Emotional" had $75.0 \%$ sensitivity and $85.7 \%$ specificity, even though only with moderate accuracy. These results suggest that VascuQOL can be a potential quantitative method to suspect the existence of CLI in dialysis PAD patients. One more advantage of VascuQOL over SPP is easy for every health professional to administer, score, and analyze without using any specific measuring machine [26].

There are still few reports to clarify the relationship between PAD and lipid markers such as apoB/apoA-I ratio, MDA-LDL, and RLP-C in dialysis patients. In this study, we examined the correlation of these atherosclerotic risk markers with PAD severity. Then, they did not show any difference between the non-CLI and CLI groups. However, all of the atherosclerotic risk markers were higher in the CLI group at the first LDL-A and 1 month after completion of LDL-A treatment. Therefore, apoB/apoA-I ratio, MDA-LDL, and RLP-C can be related to the development of PAD and the target values of preventive drug therapy for PAD in dialysis patients.

Although the present study demonstrated the possibility of VascuQOL to evaluate LDL-A treatment in dialysis patients with PAD, there are several major limitations. The main problem of this study is the lack of criterial parameter to evaluate the usefulness of VascuQOL. We have assessed VascuQOL on a speculation that it is taken for granted that PAD improve certainly after treatment of LDL-A. In order to assess the usefulness of VascuQOL for the estimation of the prolonged improvement by LDL-A treatment, it is essential to compare VascuQOL with a parameter which is well known and correlate with PAD state precisely. However, there are not a few patients whose symptom is relieved for a longer period without significant improvement of microcirculation parameters, maybe because of a number of complex local microcirculatory responses, which may contribute to rest pain and trophic changes [3, 9]. Most of the prospective study to assess the treatment of PAD select some parameters among the blood flow measurement (SPP, ABI), exercise ability (walking distance), and QOL survey (VascuQOL, SF-36) as main outcomes [28, 29]. It means that there is no gold standard method to evaluate the response to the therapy. We will need total assessment of several methods such as SPP and VascuQOL in order to evaluate the response to the therapy. Second, the present study includes a small sample size that could cause unknown source of bias in the findings. Third, the compliance to answer VascuQOL was poor. In this study, only patients who completed all the questions at all of the three evaluation points were analyzed. VascuQOL consists of simple questionnaire, but the number of questions might be large for older patients. Actually, a trial to reduce the number of questions has been already performed [26]. Several domains such as "Pain", "Symptom," and "Emotional" can be selected and assessed in the future studies to achieve better compliance.

\section{Conclusions}

We administered LDL-A treatment to 32 dialysis patients with PAD. At starting point, lipid-related arteriosclerosis markers such as apoB/apoA-I ratio, MDA-LDL, and RLP-C in the CLI group were higher than those in the non-CLI group, but not reaching a significant difference. Not only ABI, but "Symptom" and "Emotional" domains of VascuQOL were significantly higher in the CLI group. After LDL-A started, the improvement of lipid profile could not be maintained until 1 month after completion of LDL-A treatment. However, SPP improved especially at tenth LDL-A. VascuQOL improved successively until 1 month after completion of LDL-A treatment. In summary, "Symptom" and "Emotional" domains of VascuQOL can be applicable to know the severity of $\mathrm{PAD}$ in dialysis patients. LDL-A treatment induced the improvement of SPP for a long term, which caused the continuous increase of VascuQOL in dialysis patients with PAD until 1 month after completion of LDL-A treatment without changing lipid markers. VascuQOL was useful to analyze the prolonged effects of LDL-A treatment.

\section{Acknowledgements \\ The authors thank all the participants and contributors to the study. In particular, we would like to thank our collaborators: Yusuke Higashiguchi and} Rika Hanafusa, at Kawashima Dialysis Clinic.

\section{Funding}

Kenji Tsuchida has received research funds from Kaneka Corporation for other research projects.

\section{Authors' contributions}

$\mathrm{KT}$ designed and organized this experiment. KN interpreted the data, drew tables and figures, and drafted the paper. $\mathrm{MH}, \mathrm{DH}$, and $\mathrm{HM}$ collected the data. NY and DO analyzed the data. TD and JM gave us critical revisions of this article. All authors read and approved the final manuscript.

\section{Competing interests}

Kenji Tsuchida has received honoraria for speaking at events organized by Kaneka Corporation. 


\section{Consent for publication}

We have also obtained consent to publish from the participant to report individual patient data.

\section{Ethics approval and consent to participate}

All clinical investigations were conducted according to the principles expressed in the Declaration of Helsinki and individuals' data were analyzed anonymously. All patients gave their informed, written consent. This study was approved by the Research Ethics Committee of Kawashima Hospital. The registration number by the committee was 0117 .

\section{Author details}

'Department of Nephrology, Institute of Biomedical Sciences, Tokushima University Graduate School, 3-18-15, Kuramoto-cho, Tokushima-shi, Tokushima 770-8503, Japan. ${ }^{2}$ Department of Clinical Engineering, Kawashima Dialysis Clinic, 6-1, Kitasakoichiban-cho, Tokushima-shi, Tokushima 770-0011, Japan. ${ }^{3}$ Department of Kidney Disease, Kawashima Hospital, 1-39, Kitasakoichiban-cho, Tokushima-shi, Tokushima 770-0011, Japan.

Received: 5 April 2016 Accepted: 13 May 2016

Published online: 08 August 2016

\section{References}

1. Matsuzawa R, Aoyama N, Yoshida A. Clinical characteristics of patients on hemodialysis with peripheral arterial disease. Angiology. 2015;66:911-7.

2. Takahara M, lida O, Soga Y, Kodama A, Azuma N, SPINACH study investigators. Absence of preceding intermittent claudication and its associated clinical freatures in patients with critical limb ischemia. J Atheroscler Thromb. 2015;22:718-25.

3. Norgren L, Hiatt WR, Dormandy JA, Nehler MR, Harris KA, Fowkes FG, et al. Inter-society consensus for the management of peripheral arterial disease (TASC II). J Vasc Surg. 2007:45 Suppl S:S5-67.

4. Kamimura M, Matsuo M, Miyahara T, Kimura K, Matsumoto K, Nakaya T, et al. Improvements in artery occlusion by low-density lipoprotein apheresis in a patient with peripheral arterial disease. Ther Apher. 2002;6:467-70.

5. Okamoto K, Oka M, Maesato K, Ikee R, Mano T, Moriya H, et al. Peripheral arterial occlusive disease is more prevalent in patients with hemodialysis: comparison with the findings of multidetector-row computed tomography. Am J Kidney Dis. 2006:48:269-76.

6. Hoshino J, Ubara Y, Ohara K, Ohta E, Suwabe T, Higa Y, et al. Quality of life improvement and long-term effects of peripheral blood mononuclear cell transplantation for severe arteriosclerosis obliterans in diabetic patients on dialysis. Circ J. 2007;71:1193-8.

7. Kobayashi S. Applications of LDL-apheresis in nephrology. Clin Exp Nephrol. 2008;12:9-15.

8. Kobayashi S, Moriya H, Negishi K, Maesato K, Ohtake T. LDL-apheresis upregulates VEGF and IGF-I in patients with ischemic limb. J Clin Apher. 2003; 18:115-9.

9. Hara T, Kiyomoto H, Hitomi H, Moriwaki K, Ihara G, Kaifu K, et al. Lowdensity lipoprotein apheresis for haemodialysis patients with peripheral arterial disease reduces reactive oxygen species production via suppression of NADPH oxidase gene expression in leucocytes. Nephrol Dial Transplant. 2009;24:3818-25.

10. Kobayashi S, Oka M, Moriya H, Maesato K, Okamoto K, Ohtake T. LDLapheresis reduces P-Selectin, CRP and fibrinogen—possible important implications for improving atherosclerosis. Ther Apher Dial. 2006;10:219-23.

11. Tsurumi-lkeya Y, Tamura K, Azuma K, Mitsuhashi H, Wakui H, Nakazawa I, et al. Sustained inhibition of oxidized low-density lipoprotein is involved in the long-term therapeutic effects of apheresis in dialysis patients. Arterioscler Thromb Vasc Biol. 2010;30:1058-65.

12. Morimoto S, Yano Y, Maki K, Sawada K, Iwasaka T. Efficacy of low-density lipoprotein apheresis in patients with peripheral arterial occlusive disease undergoing hemodialysis treatment. Am J Nephrol. 2007;27:643-8.

13. Morgan MB, Crayford T, Murrin B, Fraser SC. Developing the Vascular Quality of Life Questionnaire: a new disease-specific quality of life measure for use in lower limb ischemia. J Vasc Surg. 2001;33:679-87.

14. de Vries M, Ouwendijk R, Kessels AG, de Haan MW, Flobbe K, Hunink MG, et al. Comparison of generic and disease-specific questionnaires for the assessment of quality of life in patients with peripheral arterial disease. J Vasc Surg. 2005;41:261-8.
15. Izquierdo-Porrera AM, Gardner AW, Bradham DD, Montgomery PS, Sorkin JD, Powell CC, et al. Relationship between objective measures of peripheral arterial disease severity to self-reported quality of life in older adults with intermittent claudication. J Vasc Surg. 2005;41:625-30.

16. Hirsch AT, Haskal ZJ, Hertzer NR, Bakal CW, Creager MA, Halperin JL, et al. ACC/ AHA 2005 guidelines for the management of patients with peripheral arterial disease (lower extremity, renal, mesenteric, and abdominal aortic): executive summary a collaborative report from the American association for vascular surgery/society for vascular surgery, society for cardiovascular angiography and interventions, society for vascular medicine and biology, society of interventional radiology, and the ACC/AHA task force on practice guidelines (writing committee to develop guidelines for the management of patients with peripheral arterial disease) endorsed by the American association of cardiovascular and pulmonary rehabilitation; national heart, lung, and blood institute; society for vascular nursing; transAtlantic inter-society consensus; and vascular disease foundation. J Am Coll Cardiol. 2006;13:1239-312.

17. Lima LM, Carvalho M, Sabino Ade P, Mota AP, Fernandes AP, Sousa MO Apo B/Apo A-I ratio in central and peripheral arterial diseases. Arq Bras Endcrinol Metabol. 2007;51:1160-5.

18. Amaki T, Suzuki T, Nakamura F, Hayashi D, Imai Y, Morita H, et al. Circulating malondialdehyde modified LDL is a biochemical risk marker for coronary artery disease. Heart. 2004;90:1211-3.

19. Witztum JL. The oxidation hypothesis of atherosclerosis. Lancet. 1994;344: 793-5.

20. Wang T, Elam MB, Forbes WP, Zhong J, Nakajima K. Reduction of remnant lipoprotein cholesterol concentrations by cilostazol in patients with intermittent claudication. Atherosclerosis. 2003;171:337-42.

21. Oishi K, Nagake Y, Yamasaki H, Fukuda S, Ichikawa H, Ota K, et al. The significance of atherogenic indices in patients on hemodialysis. Am J Nephrol. 2000;20:107-15.

22. Adorni MP, Zimetti F, Puntoni M, Bigazzi F, Sbrana F, Minichilli F, et al. Cellular cholesterol efflux and cholesterol loading capacity of serum: effects of LDL-apheresis. J Lipid Res. 2012;53:984-9.

23. Kobayashi J, Katsube S, Shimoda M, Furuhashi K, Kitano S, Masuda M, et al. Single LDL apheresis improves serum remnant-like particle-cholesterol, Creactive protein, and malondialdehyde-modified-low-density lipoprotein concentrations in Japanese hypercholesterolemic subjects. Clin Chim Acta. 2002;321:107-12.

24. Available at http://j-ca.org/wp/qol/. accessed 22 May 2016.

25. Yamaguchi T, Miyata T, Ichiki M, Inoue Y, Obitsu Y, Kichikawa K, et al. Reliability and validity of the japanese version of the Vascular Quality of Life (VascuQOL) questionnaire. J Jpn Coll Angiol. 2011;51:347-58.

26. Nordanstig J, Wann-Hansson C, Karlsson J, Lundström M, Pettersson M, Morgan MB. Vascular Quality of Life Questionnaire-6 facilitates health-related quality of life assessment in peripheral arterial disease. J Vasc Surg. 2014;59:700-7.

27. Mehta T, Venkata Subramaniam A, Chetter I, McCollum P. Assessing the validity and responsiveness of disease-specific quality of life instruments in intermittent claudication. Eur J Vasc Endovasc Surg. 2006;31:46-52.

28. Fakhry F, Spronk S, van der Laan L, Wever JJ, Teijink JA, Hoffmann WH, et al. Endovascular revascularization and supervised exercise for peripheral artery disease and intermittent claudication: a randomized clinical trial. JAMA. 2015;314:1936-44

29. Mazari FA, Carradice D, Rahman MN, Khan JA, Mockford K, Mehta T, et al. An analysis of relationship between quality of life indices and clinical improvement following intervention in patients with intermittent claudication due to femoropopliteal disease. J Vasc Surg. 2010;52:77-84.

30. Nguyen LL, Moneta GL, Conte MS, Bandyk DF, Clowes AW, Seely BL, et al. Prospective multicenter study of quality of life before and after lower extremity vein bypass in 1404 patients with critical limb ischemia. J Vasc Surg. 2006;44:977-83. discussion 983-4. 